\title{
Reliable control using the primary and dual Youla parameterizations
}

\author{
Niemann, Hans Henrik; Stoustrup, J.
}

Published in:

Proceedings of the 41st IEEE Conference on Decision and Control

Link to article, DOI:

10.1109/CDC.2002.1185057

Publication date:

2002

Document Version

Publisher's PDF, also known as Version of record

Link back to DTU Orbit

Citation (APA):

Niemann, H. H., \& Stoustrup, J. (2002). Reliable control using the primary and dual Youla parameterizations. In Proceedings of the 41st IEEE Conference on Decision and Control (Vol. 4). IEEE.

https://doi.org/10.1109/CDC.2002.1185057

\section{General rights}

Copyright and moral rights for the publications made accessible in the public portal are retained by the authors and/or other copyright owners and it is a condition of accessing publications that users recognise and abide by the legal requirements associated with these rights.

- Users may download and print one copy of any publication from the public portal for the purpose of private study or research.

- You may not further distribute the material or use it for any profit-making activity or commercial gain

- You may freely distribute the URL identifying the publication in the public portal

If you believe that this document breaches copyright please contact us providing details, and we will remove access to the work immediately and investigate your claim. 


\section{Reliable Control using the Primary and Dual Youla Parameterizations}

\author{
Henrik Niemann ${ }^{1}$
}

Abstract

Different aspects of modeling faults in dynamic systems are considered in connection with reliable control (RC). The fault models include models with additive faults, multiplicative faults and structural changes in the models due to faults in the systems.

These descriptions are considered in connection with reliable control and feedback control with fault rejection.

The main emphasis is on fault modeling. A number of fault diagnosis problems, reliable control problems, and feedback control with fault rejection problems are formulated/considered, again, mainly from a fault modeling point of view.

Reliability is introduced by means of the (primary) Youla parameterization of all stabilizing controllers, where an additional loop is closed around a diagnostic signal. In order to quantify the level of reliability, the dual Youla parameterization is introduced which can be used to analyze how large faults can be tolerated without losing e.g. stability.

\section{Introduction}

Models of faults will depend on where the fault models are going to be used. A number of faults can naturally be considered both as additive faults or as multiplicative faults. However, a random choice might not be optimal. The fault model needs to be selected with respect to the application, i.e. fault diagnosis, reliable control or feedback control with fault rejection.

In the past, additive fault models have been the most popular, especially in connection with fault diagnosis. Modeling e.g. an actuator fault as an additive fault will in general be very useful in connection with fault detection and/or fault isolation. In connection with closed-loop systems, an actuator fault might result in instability. Using an additive fault model description in this case, the fault will be considered as an external signal entering the system. The fault signal will therefore not affect the sta-

\footnotetext{
${ }^{1}$ Ørsted•DTU, Automation, Technical University of Denmark, Building 326. DK-2800 I-yngby, Denmark, Email: hhn@oersted.dtu.dk www.oersted.dtu.dk hhn

2 Dept. of Control Engineering, Aalborg University, DK 9220 Aalborg, Denmark, E-mail: jakob@ control.auc.dk www.control.auc.dk $/$ jakob
}

bility of the system. This small example indicate clearly that the description of possible faults in a dynamic system needs to be selected in very close relation with the application of the fault description/fault model.

In this paper, three types of faults/fault models will be considered. The three types are as follows:

- additive faults

- multiplicative faults - parameter faults

- system structural changes

The above fault models can be considered in connection with the following applications:

- fault detection, fault isolation and fault estimation.

- reliable control, i.e. the control system can handle faults in the system without resulting in an unstable closed loop system.

- feedback control with fault rejection, i.e. the effect from the fault is minimized in the closed loop by a fedback controller. This is equivalent with robust fiedback controller design.

It should be pointed out that this paper is not a survey paper for different reliable control methods, or of robust control methods. These areas are very well described in a large number of papers and books. Without going into details, let us mention the books by Basseville and Nikiforov [1], Gertler [5] and by Chen and Patton [4] for a good introduction to the area of fault diagnosis. The paper by Blanke et al. $[2,3]$ and by Patton $[7,8]$ and the references herein are good introductions to the area of reliable control. Most of these papers describe different concepts for RC. However, in the past years, also a number of theoretic results has been presented in this area, see e.g. $[10,12,13,14,15,17]$. The area of robust control has been investigated in a large number of books and papers. Let us only mention the books by Skogestad and Postlethwaite [9] and by Zhou et al. [16].

The focus in this paper will be on using different fault models in connection with $\mathrm{RC}$. The main results of this paper is to give an overview of what the various design problems look like depending on the type of faults. RC has already been considered in connection with additive faults in [10]. This case will therefore not be considered further in this paper. 


\section{Definitions and System Setup}

\subsection{System Setup}

Consider the following state space description of a generalized $2 \times 2$ system,

$$
\Sigma:\left\{\begin{array}{l}
e=G_{e d} d+G_{e u} u \\
y=G_{y d} d+G_{y u} u
\end{array}\right.
$$

where $d \in \mathcal{R}^{r}$ is a disturbance signal vector, $u \in \mathcal{R}^{m}$ the control input signal vector, $e \in \mathcal{R}^{q}$ is the external output signal vector to be controlled, and $y \in \mathcal{R}^{p}$ is the measurement vector

Further, let the system be controlled by a stabilizing feedback controller given by:

$$
u=K(s) y
$$

In the cases where we want to detect, isolate and/or estimate parameter changes or uncertainty variations in the system, the fault, the system can be described by:

$$
\Sigma_{M}:\left\{\begin{array}{l}
z=G_{z w} w+G_{z d} d+G_{z u} u \\
e=G_{e w} w+G_{e d} d+G_{e u} u \\
y=G_{y w} w+G_{y d} d+G_{y u} u
\end{array}\right.
$$

where $w \in \mathcal{R}^{k_{w}}$ and $z \in \mathcal{R}^{k_{2}}$ are the external input and output vectors. The connection between the external output and the external input is given by

$$
w=\Delta z
$$

where $\Delta$ represent the multiplicative faults in the system. Note that $\Delta$ can represent parametric fault as well as model uncertainties ( $\Delta=\theta$ will be applied in the following to in connection with the parametric fault case.) It should be pointed out that a direct modeling of uncertainties/parameter variations using $\Delta$ will not in general give useful results. Weighting matrices need to be included in connection with $\Delta$ for obtaining good designs. Closing the loop from $w$ to $z$ in $\Sigma_{M}$ by using $\Delta$, we get

$$
\Sigma_{\Delta}=\mathcal{F}_{u}\left(\Sigma_{M}, \Delta\right)
$$

where $\mathcal{F}_{u}(X, Y)$ is the upper Linear Fractional Transformation (LFT) of $(X, Y)$, see [16]. The lower LFT of $(X, Y)$ is given by $\mathcal{F}_{l}(X, Y)$.

Faults might change the structure of the system. One example is a sensor falling out, which will reduce the number of measurement signals. This will result in multi model systems or hybrid models. Based on a structural change of the nominal system in (1) due to faults, the system takes the following form:

$$
\Sigma_{S_{i}}:\left\{\begin{array}{c}
e=\bar{G}_{e d, i} d+\bar{G}_{e u, i} u \\
y=\tilde{G}_{y d, i} d+\tilde{G}_{y, u} u
\end{array}, i=0, \cdots, k\right.
$$

where $:$ indicates a change in the transfer function. Note that $i=0$ is defined as the nominal model, $\Sigma_{S_{0}}=\Sigma$.

\subsection{Definitions}

Based on the three different models given above, a number of definitions are now given. The definition of stable feedback control and robust feedback control are given in [16]. Reliable control is defined in [2].

Let us give three definitions in connection with feedback control and reliable control.

Definition 2.1 Given a nominal dynamic system $\Sigma$ and a feedback controller $\Sigma_{C}$. The feedback controller $\Sigma_{C}$ is said to be a stabilizing feedback controller if and only if the closed loop transfer functions of the interconnection $\mathcal{F}_{l}\left(\Sigma, \Sigma_{c}\right)$ is internally stable.

Definition 2.2 Given a dynamic system $\Sigma_{\Delta}$ and a feedback controller $\Sigma_{C}$. It is assumed that $\Delta \in \Delta$ represents the model uncertainty. The feedback controller $\Sigma_{C}$ is said to be a robustly stabilizing feedback controller if and only if the closed loop transfer functions of the interconnection $\mathcal{F}_{l}\left(\Sigma_{\Delta}, \Sigma_{c}\right)$ is internally stable for all $\Delta \in \underline{\Delta}$.

Definition 2.3 Given a set of dynamic systems $\Sigma_{S_{i}}, i=$ $0, \cdots, k$, and a feedback controller $\Sigma_{C}$. The feedback controller $\Sigma_{C}$ is said to be a reliable feedback controller if and only if the closed loop transfer functions of the interconnection $\mathcal{F}_{l}\left(\Sigma_{S_{i}}, \Sigma_{c}\right), i=0, \cdots, k$ is intemally stable.

\section{Reliable Control}

Just as in connection with fault diagnosis, the reliable control problem will depend strongly on the type of faults that can appear in the system. In this paper, the various reliable control design problems will be described for the three different model structures given in Section 2. Especially in connection with RC for systems with structural changes, the solution (the selected controller structure, type etc.) will depend strongly on the specific case. There does not exist any general method with explicit design formulae that can handle the general case. Much better design results can be obtained by using dedicated design methods.

\subsection{The Youla Parameterization}

Before considering the three different RC design cases, the (primary) Youla parameterization is shortly introduced. The Youla parameterization will be applied in connection with $\mathrm{RC}$ in the following. The Youla parameterization has also been applied in connection with RC in $[10,17]$.

Let a coprime factorization of the system $G_{y u}(s)$ from (1) and a stabilizing controller $K(s)$ from (2) be 
given by:

$$
\begin{array}{lr}
G_{y u}=N_{u} M^{-1}=\tilde{M}^{-1} \tilde{N}_{u}, & N_{u}, M, \tilde{N}_{u}, \tilde{M} \in \mathcal{R} \mathcal{H}_{\infty} \\
K=U V^{-1}=\bar{V}^{-1} \tilde{U}, & U, V, \tilde{U}, \tilde{V} \in \mathcal{R} \mathcal{H}_{\infty}
\end{array}
$$

where the eight matrices in (6) must satisfy the double Bezout equation given by, see [16]:

$$
\begin{aligned}
\left(\begin{array}{ll}
I & 0 \\
0 & I
\end{array}\right) & =\left(\begin{array}{cc}
\tilde{V} & -\tilde{U} \\
-\tilde{N}_{u} & \tilde{M}
\end{array}\right)\left(\begin{array}{cc}
M & U \\
N_{u} & V
\end{array}\right) \\
& =\left(\begin{array}{cc}
M & U \\
N_{u} & V
\end{array}\right)\left(\begin{array}{cc}
\tilde{V} & -\tilde{U} \\
-\bar{N}_{u} & \tilde{M}
\end{array}\right)
\end{aligned}
$$

Based on the above coprime factorization of the system $G_{y u}(s)$ and the controller $K(s)$, we can give a parameterization of all controllers that stabilize the system in terms of a stable parameter $Q(s)$, i.e. all stabilizing controllers are given by [11]:

$$
K(Q)=U(Q) V(Q)^{-1}
$$

where

$$
U(Q)=U+M Q, V(Q)=V+N_{u} Q, Q \in \mathcal{R} \mathcal{H}_{\infty}
$$

or by using a left factored form:

$$
K(Q)=\tilde{V}(Q)^{-1} \tilde{U}(Q)
$$

where

$$
\tilde{U}(Q)=\tilde{U}+Q \tilde{M}, \tilde{V}(Q)=\tilde{V}+Q \tilde{N}_{u}, Q \in \mathcal{R} \mathcal{H}_{\infty}
$$

Using the Bezout equation, the controller given either by (8) or by (9) can be realized as an LFT in the parameter $Q$,

$$
K(Q)=\mathcal{F}_{l}\left(J_{K}, Q\right)
$$

where $J_{K}$ is given by

$$
J_{K}=\left(\begin{array}{cc}
U V^{-1} & \tilde{V}^{-1} \\
V^{-1} & -V^{-1} N_{u}
\end{array}\right)
$$

Introducing the transfer function from the disturbance signal $d$ output $y$ given by $G_{y d}$ from (1) in connection with the coprime factorization of $G_{y u}$ in (6), we obtain the following relationship:

$$
y=\left(\begin{array}{ll}
G_{y d} & G_{y u}
\end{array}\right)\left(\begin{array}{l}
d \\
u
\end{array}\right)=\tilde{M}^{-1}\left(\begin{array}{ll}
\tilde{N}_{d} & \tilde{N}_{u}
\end{array}\right)\left(\begin{array}{l}
d \\
u
\end{array}\right)
$$

Reorganizing the controller $K(Q)$ given by (10) results in the closed loop system depicted in Figure 1, [11].

The main observation which shall be exploited in the solution to the reliabie control problem, is the following very simple expression for the transfer function from faults to measurements in terms of the parameter $Q$ :

$$
\begin{aligned}
y & =\tilde{M}^{-1}\left(\tilde{N}_{d} d+\tilde{N}_{u} u\right) \\
& =\left(V+N_{u} Q\right) \tilde{N}_{d} d
\end{aligned}
$$

where (7) has been exploited.

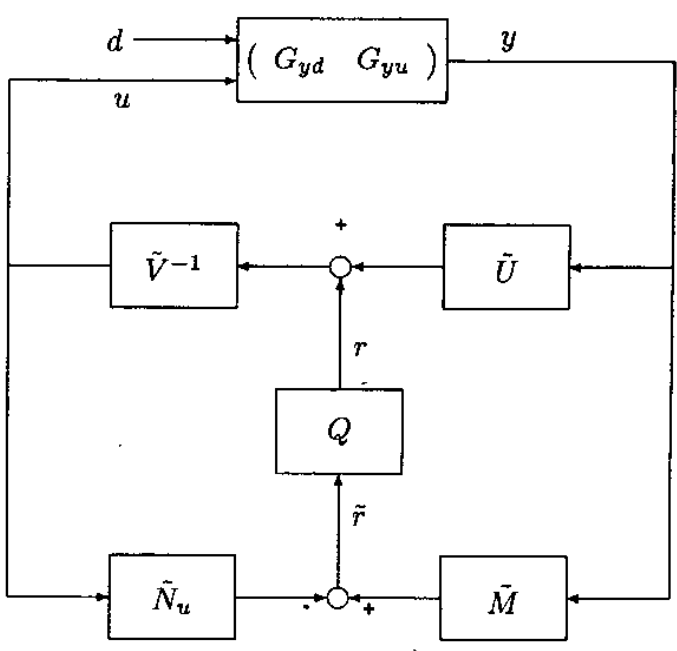

Figure 1: Controller structure with parameterization

\subsection{RC for Systems with Multiplicative Faults}

In this case, the closed-loop stability can be affected by the multiplicative faults, if $G_{y u}$ depend on the multiplicative faults. A Youla parameterized controller $K(Q)$ is applied, where the nominal controller $K(0)=K_{0}$ is designed for the nominal system. The Youla parameter is then applied for obtaining RC, i.e. $Q$ needs to stabilize the closed-loop system when a fault has appeared in the system. The stability of the closed loop system requires stability of the nominal closed-loop system and closed-loop stability of a loop where both $Q$ and the multiplicative faults $\Delta$ are included, [11]. The stability of the closed-loop system is satisfied by the design of the nominal feedback controller $K(0)$. The other closedloop system that needs to be stable is given by

$$
\bar{S}(Q)=(I-Q S(\Delta))^{-1}
$$

where $S(\Delta)$ is the dual Youla parameter, [11], depending on the multiplicative faults $\Delta$. The dual Youla parameter $S$ is a parameterization of all systems stabilized by a given controller $K(0),[11]$. The dual Youla parameter $S$ or $\tilde{S}$ can be directly estimated from known signals. Consider the system setup in Figure 1, the open loop transfer function from $r$ to $\tilde{r}$ is the transfer function $S,[10,11]$ and the closed loop transfer function $\bar{S}$ is the associated closed loop transfer function. This is important, because it makes it possible to estimate $S$ or $\tilde{S}$ which are measures for the faults in the system.

It is required that $S$ is stable to guarantee closed-loop stability. Combining the Youla parameterization with the dual Youla parameterization, it is not a condition that $Q$ and $S$ need to be stable to guarantee closed-loop stability. $Q$ and $S$ just need to satisfy that the closed-loop system given by (12) is stable, [11]. $S(\Delta)$ take the following form in the general case:

$S(\Delta)=\tilde{M} G_{y w} \Delta\left(I-\left[G_{z w}+G_{z u} U \bar{M} G_{y w}\right] \Delta\right)^{-1} G_{z u} M$ 
In connection with (13), it is important to note that the stability condition of $S$ and/or of $\bar{S}(Q)$ in (12) for satisfying that the faulty closed loop system is stable, is only valid if the faulty system is still detectable and stabilizable from the specified input signals $u$ and output signals $y$. This is a standard condition in connection with RC systems. If the faulty system is not detectable and/or stabilizable, additional actuators and/or sensors need to be included in the system to satisfy these two conditions. It should be pointed out that the RC setup considered in this paper, does not restrict the possibility to include more general controller architectures, where the number of actuators and/or sensors can be changed/modified in connection with faults. This subject, however, will not be described in this paper.

In the general case, the equation for $S(\Delta)$ given above is quite complicated. $S(\Delta)$ needs to be derived explicitly in every single case in order to reduce the complexity of $S(\Delta)$. Consider two simple cases, where the multiplicative faults are placed at either the input to the system (actuator faults) or at the output to the system (sensor faults), i.e. the system given by (3) takes the following form

$$
\left(\begin{array}{ll}
G_{e d}(\Delta) & G_{e u}(\Delta) \\
G_{y d}(\Delta) & G_{y u}(\Delta)
\end{array}\right)=\left(\begin{array}{ll}
G_{e d} & G_{e u}+G_{e u} \Delta \\
G_{y d} & G_{y u}+G_{y u} \Delta
\end{array}\right)
$$

for multiplicative faults at the input. The system given by (3) takes the following form for multiplicative faults at the output

$$
\left(\begin{array}{cc}
G_{e d}(\Delta) & G_{e u}(\Delta) \\
G_{y d}(\Delta) & G_{y u}(\Delta)
\end{array}\right)=
$$

The dual Youla parameter $S$ is then given by:

$$
S(\Delta)=\tilde{N}_{u} \Delta\left(I-U \tilde{N}_{u} \Delta\right)^{-1} M
$$

for multiplicative faults at the input and

$$
S(\Delta)=\tilde{M}_{u} \Delta\left(I-N_{u} \tilde{U} \Delta\right)^{-1} N_{u}
$$

for multiplicative faults at the output.

It is important to note that if $S$ is stable, we do not need a $Q$-parameter to stabilize the system. In this way, $S$ can be used for analyzing which faults are admissible and how large they can be before the closed-loop system will become unstable.

In Table 1, $S$ has been calculated for a number of different types of multiplicative faults.

So far, the stability part with respect to multiplicative faults has been treated. This is the most important part of the RC. However, it will also in some cases be possible to design the $\mathrm{RC}$ controller (the $Q$ controller) with respect to both closed-loop stability as well as closedloop performance. Closing the loop of the system in (3) with the feedback controller $K(Q)$, we get the following closed loop transfer function, see [6]

$$
e=T_{e d}(s) d
$$

where

$$
\begin{aligned}
& T_{e d}(s)=G_{e d}(\Delta)+G_{e u}(\Delta)(U+M Q) \\
& \times\left(\left(V-G_{y u}(\Delta) U\right)+\left(N_{u}-G_{y u}(\Delta) M\right) Q\right)^{-1} G_{y d}
\end{aligned}
$$

with

$$
\begin{aligned}
& G_{e d}(\Delta)=G_{e d}+G_{e w} \Delta\left(I-G_{z w} \Delta\right)^{-1} G_{z d} \\
& G_{e u}(\Delta)=G_{e u}+G_{e w} \Delta\left(I-G_{z w} \Delta\right)^{-1} G_{z u} \\
& G_{y d}(\Delta)=G_{y d}+G_{y w} \Delta\left(I-G_{z w} \Delta\right)^{-1} G_{z d} \\
& G_{y u}(\Delta)=G_{y u}+G_{y w} \Delta\left(I-G_{z w} \Delta\right)^{-1} G_{z u}
\end{aligned}
$$

Now, let us again consider the two cases with multiplicative input faults and output faults. The general system in (3) is then given by (14) and (15), respectively. The general closed loop transfer function in (18) is given by

$$
\begin{aligned}
& T_{e d}(\Delta)=G_{e d}+G_{e u}(I+\Delta)(U+M Q) \\
& \left(I-\bar{N}_{u} \Delta(U+M Q)\right)^{-1} \bar{M} G_{y d}
\end{aligned}
$$

for multiplicative faults at the input and

$$
\begin{aligned}
& T_{e d}(\Delta)=G_{e d}+G_{e u} M(\tilde{U}+Q \tilde{M}) \tilde{M} \\
& \left(I-\Delta N_{u}(\tilde{U}+Q \tilde{M})\right)^{-1}(I+\Delta) G_{y d}
\end{aligned}
$$

for multiplicative faults at the output, respectively.

Using a standard setup formulation, we get the following open loop transfer functions for the design of the $Q$ controller in the two cases (see Figure 2 for the standard setup). For the input fault case, we have

$$
P(\Delta)=\left(\begin{array}{cc}
\tilde{G}_{e d}(\Delta) & \bar{G}_{e u}(\Delta) \\
\tilde{G}_{y d}(\Delta) & \tilde{G}_{y u}(\Delta)
\end{array}\right)
$$

where

$$
\begin{aligned}
\tilde{G}_{e d}(\Delta) & =G_{e d} \\
& +G_{e u}(I+\Delta) U\left(I-\tilde{N}_{u} \Delta U\right)^{-1} \tilde{M} G_{y d} \\
\tilde{G}_{e u}(\Delta) & =G_{e u}(I+\Delta)\left(I-U \tilde{N}_{u} \Delta\right)^{-1} M \\
\tilde{G}_{y d}(\Delta) & =\left(I-\tilde{N}_{u} \Delta U\right)^{-1} \tilde{M} G_{y d} \\
\tilde{G}_{y u}(\Delta) & =\left(I-\bar{N}_{u} \Delta U\right)^{-1} \tilde{N}_{u} \Delta M
\end{aligned}
$$

and for the output fault case, we have

$$
P(\Delta)=\left(\begin{array}{cc}
\tilde{G}_{e d}(\Delta) & \tilde{G}_{e u}(\Delta) \\
\tilde{G}_{y d}(\Delta) & \tilde{G}_{y u}(\Delta)
\end{array}\right)
$$

where

$$
\begin{aligned}
\tilde{G}_{e d}(\Delta) & =G_{e d} \\
& +G_{e u} M \tilde{U}\left(I-\Delta N_{u} \tilde{U}\right)^{-1}(I+\Delta) G_{y d} \\
\tilde{G}_{e u}(\Delta) & =G_{e u} M\left(I-\tilde{U} \Delta N_{u}\right)^{-1} \\
\tilde{G}_{y d}(\Delta) & =\bar{M}\left(I-\Delta N_{u} \tilde{U}\right)^{-1}(I+\Delta) G_{y d} \\
\tilde{G}_{y u}(\Delta) & =\tilde{M} \Delta N_{u}\left(I-\tilde{U} \Delta N_{u}\right)^{-1}
\end{aligned}
$$

respectively.

It is possible to combine reliable control with fault isolation. It is then possible to design a number of $Q$ controllers, one for every single fault case and then select a specific $Q$ controller when a fault appear in the system. 


\begin{tabular}{|c|c|}
\hline System description, $G_{y u}(\Delta)$ & The dual Youla parameter, $S(\Delta)$ \\
\hline$G_{y u}(\Delta)=(I+\Delta) G_{y u}$ & $S(\Delta)=\tilde{M} \Delta(I-N \tilde{U} \Delta)^{-1} N$ \\
\hline$G_{y u}(\Delta)=G_{y u}(I+\Delta)$ & $S(\Delta)=\tilde{N} \Delta(I-U \tilde{N} \Delta)^{-1} M$ \\
\hline$G_{y u}(\Delta)=G_{y u}+\Delta$ & $S(\Delta)=\tilde{M} \Delta(I-U \tilde{M} \Delta)^{-1} M$ \\
\hline$G_{y u}(\Delta)=G_{y u}(I+\Delta)^{-1}$ & $S(\Delta)=-\tilde{N} \Delta(I+M \tilde{V} \Delta)^{-1} M$ \\
\hline$G_{y u}(\Delta)=(I+\Delta)^{-1} G_{y u}$ & $S(\Delta)=-\tilde{M} \Delta(I+V \tilde{M} \Delta)^{-1} N$ \\
\hline$G_{y u}(\Delta)=G_{y u}\left(I+\Delta G_{y u}\right)^{-1}$ & $S(\Delta)=-\tilde{N} \Delta(I+N \tilde{V} \Delta)^{-1} N$ \\
\hline$G_{y u}(\Delta)=\left(N+\Delta_{N}\right)\left(M+\Delta_{M}\right)^{-1}$ & $S(\Delta)=\left(\begin{array}{c}-\tilde{M})\left(\Delta_{\Delta}^{M}\right)\left(I+(V-U)\left(\Delta_{M}^{M}\right)\right)^{-1} \\
\hline G_{y u}(\Delta)=\left(\tilde{M}+\Delta_{\tilde{M}}\right)^{-1}\left(\tilde{N}+\Delta_{\tilde{N}}\right)\end{array}\right.$ \\
\hline
\end{tabular}

Table 1: The connection between different system multiplicative faults in terms of $\Delta$ and the dual Youla parameter $S$.

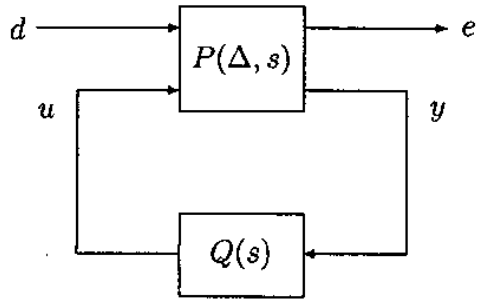

Figure 2: The standard setup for design of $Q$ for systems with multiplicative faults

\subsection{RC for Systems with Structural Changes}

This is the most relevant problem in connection with RC. From a feedback point of view, a fault in a closed-loop system will in most cases change the structure of the system. However, in many cases, these structural changes can be described by using LFTs as considered in the multiplicative fault case.

In the following, let us just consider the system given by transfer functions described by (5). It is further assumed that the system can only be in the normal (nominal) mode and in one abnormal mode. The abnormal mode is given by:

$$
\Sigma_{S}:\left(\begin{array}{cc}
\tilde{G}_{e d} & \tilde{G}_{e u} \\
\bar{G}_{y d} & \tilde{G}_{y u}
\end{array}\right)
$$

The closed loop transfer function for the nominal system $\Sigma$ and $\Sigma_{S}$ when the feedback controller in (2) is applied are given by

$$
\begin{gathered}
T_{e d, n o m}(s)=G_{e d}+G_{e u} K\left(I-G_{y u} K\right)^{-1} G_{y d} \\
T_{e d, S}(s)=\tilde{G}_{e d}+\tilde{G}_{e u} K\left(I-\tilde{G}_{y u} K\right)^{-1} \tilde{G}_{y d}
\end{gathered}
$$

From the closed loop transfer functions in (24), it is clear that it is not possible to do RC if the change is in $G_{e d}$ and/or $G_{e u}$. For doing RC, we need to observe/detect the change. Instead, if there is also a change in $G_{y u}$ and/or $G_{y d}$ at the same time, we might be able to make $\mathrm{RC}$ for the system.

Following the line from the above section, we can again calculate $S$ as a function of the system changes and use this for obtaining RC. The structural changes of $G_{y u}$ can be described in the following way:

$$
\begin{aligned}
\tilde{G}_{y u} & =G_{y u}+\left(\tilde{G}_{y u}-G_{y u}\right) \\
& =G_{y u}+\tilde{\Delta}
\end{aligned}
$$

From Table 1, we have that

$$
S=\tilde{M} \tilde{\Delta}(I-U \tilde{M} \tilde{\Delta})^{-1} M
$$

Using $\tilde{\Delta}=\tilde{G}_{y u}-G_{y u}$ in $S$, we get directly

$$
S=\left(\tilde{M} \tilde{G}_{y u}-\bar{N}_{u}\right)\left(\bar{V}-\tilde{U} \tilde{G}_{y u}\right)^{-1}
$$

$Q$ needs again to be designed such that

$$
\tilde{S}(Q)=(I-Q S)^{-1}
$$

is stable.

As a direct result of the above analysis, is that systems with structural changes can also be handled as systems with multiplicative faults. Therefore no further results will be given here. 


\section{Conclusion}

In this paper a common framework based on linear fractional transformations has been introduced, which facilitates modeling of additive faults, multiplicative faults, as well as faults that change the model structure.

By applying the (primary) Youla parameterization, an additional controller parameter has been introduced as the main tool to achieve reliability. A feature of the Youla parameterization is that it automatically includes a diagnostic signal.

In order to quantify the reliability of a given configuration, the dual Youla parameterization has been introduced. The corresponding parameter reflects how large faults can be handled by the RC system without losing e.g. stability or performance.

Although faults leading to structural changes of a system in principle calls for ad hoc solutions, it has still been possible to give general formulae for fairly rich and important classes of structural changes. An example can be found in [6].

One aspect that has not been considered in this paper is the synthesis of the $Q$ controller with respect to both the nominal case as well as the faulty case. The focus in this paper has only been on the design of $Q$ with respect to some possible faults in the system. However, if we let the $Q$ controller also be active in the nominal case, the design of $Q$ turns out to be a multi objective design. The design of $Q$ then needs to be done with respect to a disturbance rejection in the nominal case and with respect to closed loop stability in the faulty case. The disturbance rejection design is an open-loop design of $Q$. This aspect has been considered further in [6].

\section{References}

[1] M. Basseville and I.V. Nikiforov. Detection of abrupt changes - theory and application. Prentice Hall, 1993.

[2] M. Blanke, C.W. Frei, F. Kraus, R.J. Patton, and M. Staroswiecki. What is fault-tolerant control? In Preprints of 4th IFAC Symposium on Fault Detection Supervision ans Safety for Technical Processes, SAFEPROCESS'2000, pages 40-51, Budapest, Hungary, 2000.

[3] M. Blanke, M. Staroswiecki, and E. Wu. Concepts and methods in fault-tolerant control. In Proceedings of American Control Conference, ACC-2001, pages 26062620, Washington DC, USA, 2001.

[4] J. Chen and R. Patton. Robust model-based fault diagnosis for dynamic systems. Kluwer Academic Publishers, 1998.

[5] J. Gertler. Fault detection and diagnosis in engineering systems. Marcel Dekker, 1998.
[6] H. Niemann and J. Stoustrup. An architecture for reliable controllers. Submitted for publication, 2002.

[7] R. Patton. Fault tolerant control: The 1997 situation. In Proceedings of the IFAC Symposium SAFEPRO. CESS'97, pages 1033-1055, Hull, England, 1997.

[8] R. Patton. Where are we in fault tolerant control. Seminar notes, June 1997. Centre for Systems and Control, Faculty of Engineering, Glasgow University, Glasgow, G12 8QQ, UK.

[9] S. Skogestad and I. Postlethwaite. Multivariable feedback control - Analysis and design. John Wiley \& Sons, 1996.

[10] J. Stoustrup and H.H. Niemann. Fault tolerant feedback control using the youla parameterization. In Proceedings of the 6th European Control Conference, Porto, Portugal, September 2001.

[11] T.T. Tay, I.M.Y. Mareels, and J.B. Moore. High performance control. Birkhäuser, 1997.

[12] Y.Y. Wang and N.E. Wu. An approach to configuration of robust control systems for robust failure detection. In Proceedings of the 32 nd Conference on Decision and Control, pages 1704-1709, San Antonio, Texas, USA, 1993.

[13] N.E. Wu. Reconfigurable control design: Achieving stability robustness and failure tracking. In Proceed ings of the 32nd Conference on Decision and Control, pages 2278-2283, San Antonio, Texas, USA, 1993.

[14] N.E. Wu and T.J. Chen. Feedback design in control reconfigurable systems. International Journal of Robust and Nonlinear Control, 6(6):561-570, 1996.

[15] N.E. Wu, K. Zhou, and G. Salomon. Control reconfigurability of linear time-invariant systems. Automatica, 36:1767-1771, 2000.

[16] K. Zhou, J.C. Doyle, and K. Glover. Robust and optimal control. Prentice Hall, 1995.

[17] K. Zhou and Z. Ren. A new controller architecture for high performance robust, and fault-tolerant control. IEEE Transactions on Automatic Control, 46(10):1613$1618,2001$. 